Review Article

\title{
PHARMACEUTICAL AND BIOPHARMACEUTICAL ASPECTS OF QUANTUM DOTS-AN OVERVIEW
}

\section{SADDAM C. SHAIKH ${ }^{1}$, SHWETA G. SABO0 ${ }^{2}$, PRASHANT S. TANDALE ${ }^{3}$, FAHIM S. MEMON ${ }^{4}$, SHARAD D. TAYADE ${ }^{*}$, M. AKIFUL HAQUE ${ }^{5}$, SHARUK L. KHAN ${ }^{6}$}

${ }^{1}$ Department of Pharmaceutics (D Pharm), Rajarshi Shahu College of Pharmacy, Buldana, Maharashtra, India 443001, ${ }^{2}$ Department of Pharmacognosy, Government College of Pharmacy, Karad, Maharashtra, India 415124, ${ }^{3}$ Department of Pharmaceutics, School of Pharmaceutical Sciences, Lovely Professional University, Punjab, India 144001, ${ }^{4}$ Department of Pharmaceutics, Rajarshi Shahu College of Pharmacy, Buldana, Maharashtra, India-443001, ${ }^{5}$ Department of Pharmaceutical Analysis, Anurag University, Venkatapur, Hyderabad, India 500088, ${ }^{\circ}$ Department of Pharmaceutical Chemistry, MUP's College of Pharmacy (B Pharm), Degaon, Risod, Washim, Maharashtra, India 444504 Emails: sharad_tayade1@rediffmail.com

Received: 23 Mar 2021, Revised and Accepted: 12 May 2021

\section{ABSTRACT}

In the twenty-first century, nanotechnology has become cutting-edge technology. It is interdisciplinary and multidisciplinary, covering numerous fields such as medicine, engineering, biology, physics, material sciences, and chemistry. The present work aims to cover the optical properties, method of preparations, surface modifications, bio-conjugation, characterization, stability, and cytotoxicity of quantum dots (QDs).

Articles were reviewed in English literature reporting the pharmaceutical and bio-pharmaceutical aspects of QDs which were indexed in Scopus, web of science, google scholar and PubMed without applying the year of publication criterion.

One significant value of utilizing nanotechnology is that one can alter and control the properties in a genuinely unsurprising way to address explicit applications' issues. In science and biomedicine, the usage of functional nanomaterials has been broadly investigated and has become one of the quick-moving and stimulating research directions. Different types of nanomaterial (silicon nanowires, QDs, carbon nanotubes, nanoparticles of gold/silver) were extensively utilized for biological purposes. Nanomedicine shows numerous advantages in the natural characteristics of targeted drug delivery and therapeutics. For instance, protection of drugs against degradation, improvement in the drug's stability, prolonged circulation time, deceased side effects, and enhanced distribution in tissues. The present review article deals with the quantum dots, their optical properties, method of preparations, surface modifications, bio-conjugation, characterization, stability, and cytotoxicity of quantum dots. The review also discusses various biomedical applications of QDs.

The QDs-based bio-nanotechnology will always be in the growing list of unique applications, with progress being made in specialized nanoparticle development, the detection of elegant conjugation methods, and the discovery of new targeting ligands.

Keywords: Quantum dots, Optical properties, Microwave-assisted method, Cytotoxicity, Cell imaging, Sentinel lymph-node mapping

(C) 2021 The Authors. Published by Innovare Academic Sciences Pvt Ltd. This is an open access article under the CC BY license (https://creativecommons.org/licenses/by/4.0/] DOI: https://dx.doi.org/10.22159/ijap.2021v13i4.41623. Journal homepage: https://innovareacademics.in/journals/index.php/ijap

\section{INTRODUCTION}

In 1981, Ekimov and Onushenko described the Quantum dots (QDs) also called nanoscale semiconductor crystals [1]. The quantum dots are made up of material from periodic table group II-VI (CdSe) or III$\mathrm{V}$ (InP) [2-6]. The most well studied and broadly utilized QDs are the cadmium selenide $(\mathrm{Cd} / \mathrm{Se})[3]$. The cores and QDs targeting are protected by surface modification, and such changes significantly improve QDs sizes [7]. There are two kinds of fluorescent-based quantum dots, namely graphene and carbon quantum dots [8]. Quantum dots possess quantum confinement property and, on excitation from visible to infra-red wavelength, emit fluorescence [9, 10]. Usually, in the crystal core of a single QD, around 100-100,000 atoms are present. The size of QDs usually lies in between $2-10 \mathrm{~nm}$ diameter, which generally grows up to $5-20 \mathrm{~nm}$ in diameter after encapsulation of polymer [11,12]. QDs comprise a semiconductor core, which is over-covered by shell and cap. The anatomy of QDs is represented in fig. 1 [13]. Semiconducting character and optical properties depend on the inorganic core. In QDs synthesis, the organic surfactants are developed and eventually shape ligands on the core surface [14-17]. The nature of the capping agent produced significantly relies on the final application of QDs. Frequently used ligands in QDs synthesis are alcohols, essential amines, and thiols $[18,19]$. Bio-conjugation with carbohydrates, viruses, natural products, DNA fragments, and peptides assist by ligands through the covalent coupling and electrostatic or hydrophobic interactions [20 21]. Determination of solubility, colloidal stability, control particle morphology, particle size distribution, and accumulation chiefly depends on ligands [22-24]. The present work aims to cover the optical properties, method of preparations, surface modifications, bio-conjugation, characterization, stability, and cytotoxicity of QDs. Articles were reviewed in English literature reporting the pharmaceutical and bio-pharmaceutical aspects of QDs which were indexed in Scopus, web of science, google scholar and PubMed without applying the year of publication criterion. The keywords used for searching the literature are quantum dots, quantum dots in drug delivery system, methods of preparation of quantum dots, applications of quantum dots, recent advances in quantum dots, pharmaceutical and biopharmaceutical applications of quantum dots etc.

QDs core consists of several substances like cadmium, zinc, lead chalcogenides (CdS, CdSe, CdTe), copper salt $(\mathrm{CuCl})$, arsenides (InAs, GaAs), semiconducting phosphides (InP, GaP), and nitrides (GaN). The shell of CdSe, $\mathrm{ZnSe}, \mathrm{PbS}, \mathrm{ZnS}, \mathrm{ZnTe}, \mathrm{CdS}$, ZnO generally enclosed the core [3]. The core of nanomaterials guarded by outer covering from photoinitiated degradation and removing surface defects enhances the luminescence properties. Besides, cadmium, silver, copper, manganese, and rare earth metal ions can be used to dope the core of nanocrystals to enhance photoluminescence properties $[25,26]$. QDs can be assembled, permitting particle shape, size, and chemical composition to be changed by suit a given application. The design and size of QDs are the properties that are frequently manipulated; this will decide if the QDs are chemically excited in NIR or UV light [27-29]. The biological targeting molecules (e. g., antibodies) or biocompatible polymeric materials (e. g., PEG) can be functionalized on the amenable external surface of QDs to improve their physiological system performance. The most commonly utilized QDs consist of graphene QDs, carbon QDs, and cadmiumbased QDs [30-32]. QDs are found to be photochemically stable with symmetric, narrow, and strong fluorescence emission. QDs are 
perfectly suitable for repetitive measurements or long-term observations [33-35]. After intradermal administration of dots in mouse paw, the near IR emitting dots might be noticed in the intraoperative imaging system's lymphatic system [36-39]. A report proposed that probes of quantum rods conjugated with transferring were productive for in vitro blood-brain barrier transmigration [40-43].
In fig. 2 composition of QDs is mentioned along with the material used [44]. The QDs are metastable and generally, through chemical surface modification, need to be stabilized. QDs show narrow-size tunable emission spectra, high extinction coefficients, and much diminishing photobleaching rates compared to organic dyes [45-48].

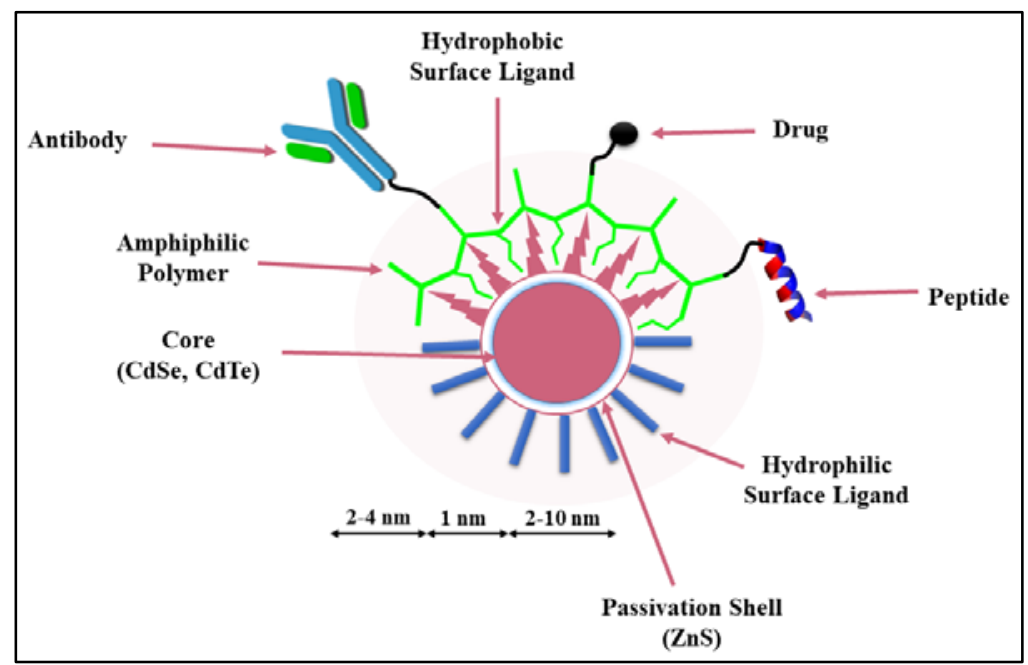

Fig. 1: The anatomy of QDs

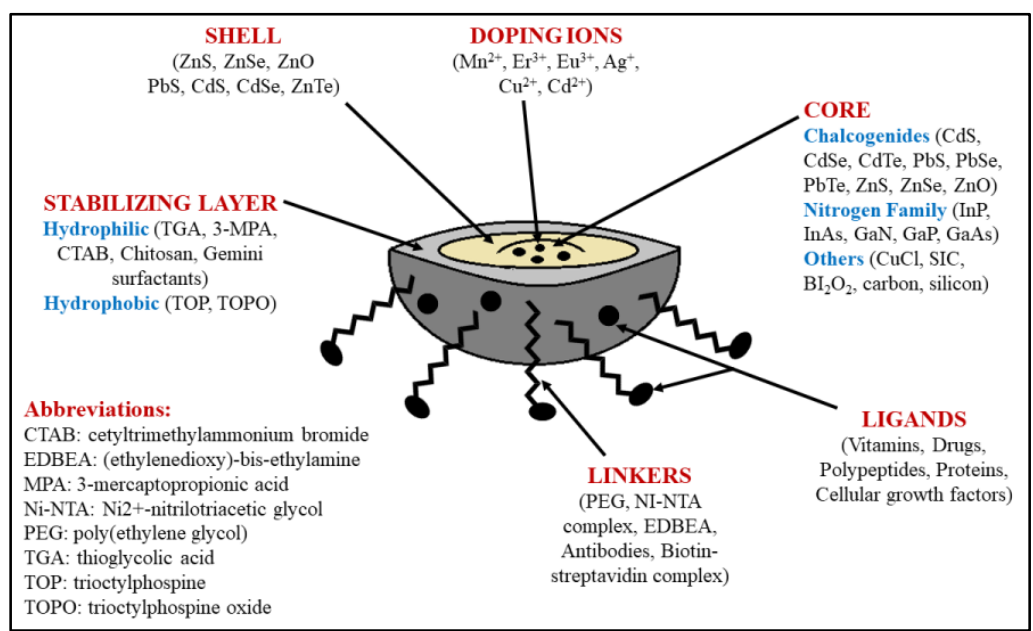

Fig. 2: The composition and material used for the synthesis of QDs

\section{Advantages of QDs}

QDs possess good physical stability and speedy degradation observed in optical imaging probes compared to QDs, whereas QDs depicted high instead of degradation. As compared to traditional dyes, QDs comprised higher photostability due to their fluorescence intensity and unique inorganic composition. QDs have a narrow emission peak and broader excitation spectra, whereas sharp emission peaks are depicted by organic dyes and narrow absorption spectrum observed with organic fluorophores. Due to these features, less energy is sufficient for the excitation of QDs irrespective of size. Hence, to excite multi-color QDs, single ultraviolet or blue wavelength beam is adequate. As compared to organic dyes, QDs show considerably longer fluorescence lifetimes and are 10-20 times brighter. Using a single source, QDs can feasibly be excited, and concurrently several targets tracked in vivo with the application of various probes as permitted by the multi-color QDs. With low background interference, high signal intensity depicts by the QDs conjugates due to sharp emission spectra and large Stokes Shift. QDs can be molded into various shapes, such as quantum dust, small crystals, and bead forms, and can be coated with different biomaterials. QDs have comfortable and cost-effective manufacturing methods. Colloidal synthesis, epitaxial techniques, and lithographic techniques are various manufacturing methods of QDs [49-53].

\section{Disadvantages of QDs}

In biomedical applications, the size of the QDs has terrific importance. Through renal filtration, QDs having small size get removed from the body easily; however, there may be chances that the reticuloendothelial system takes the larger particles before reaching the targeted disease sites. The optimal activity is demonstrated by QDs having a 5-20 nm size of the polymer coating. When located in live cells, QDs aggregate and may interferes with cell function or kill the cells. Bio-conjugation of QDs results in difficult delivery of QDs into the target. QDs have unknown metabolism and excretion, leading to toxicity if it accumulates in the body tissues, spleen, liver and kidney. QDs become toxic if coated with mercaptoacetic acid. Even building material or the core of QDs (CdSe) is also toxic. Heavy metal ions 
leak from the core due to oxidation and photolysis, which results in cytotoxicity $[49,50,54-57]$.

\section{Optical properties}

QDs have expanded consideration because of their exceptional optical properties compared to traditional fluorescent dyes [58-60]. The distinctive optical properties of QDs permit multi-color imaging with no cross-talk in fluorescence microscopes among various detection channels. Moreover, with one single wavelength QDs having different emission maxima is excite and demand for many excitation sources is reduced. Time-resolved detection is possible because of the comparatively long fluorescence lifetime of the QDsfluorescence, which considerably enhances the ratio (by 15 factors) of signal-to-background concerning cell autofluorescence. QDs emission maxima can be correctly adjusted by changing QDs size. Short-wavelength light emits by smaller QDs as compared to larger particles $[58,60]$. Electronic and unique optical properties in QDs are due to quantum confinement effects offering various benefits on current fluorophores, like lanthanide chelates, fluorescent proteins, and organic dyes. Properties that significantly impact fluorophore behavior and subsequently relevance to multiple circumstances consist of photostability, the width of the emission spectrum, decay lifetime, and the excitation spectrum. Concurrently, various QDs can emit different colors beneath the same excitation light to track and image numerous molecular targets [28-31]. The multi-color QDs probes can be utilized at the same time, as shown in fig. 3 [61].

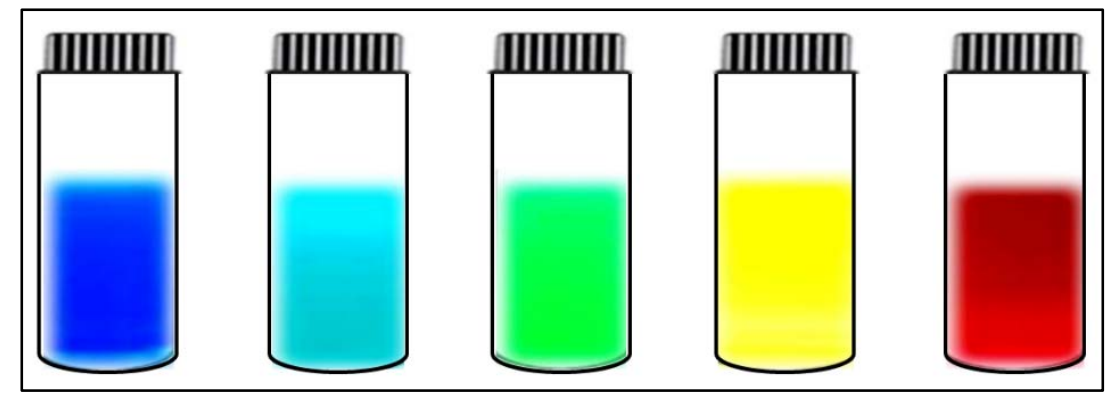

Fig. 3: In the visible range at various wavelengths, QDs displaying hues of colors

\section{Methods of preparation}

QDs are manufactured by various methods like chemical precipitation method, polyol-hydrolysis method, $\gamma$-radiation rout, electron beam irradiation, photochemical synthesis $[35,36,45,47$, 62-68]. However, few techniques of QDs fabrication are discussed below.

\section{Organic phase method/organometallic method}

Monodispersed QDs can be prepared through the organometallic process. Using this method, one can prepare monodispersed QDs with regularity in the core structure with uniform surface derivatization. Bis(trimethylsilyl) selenium and $\mathrm{Me}_{2} \mathrm{Cd}$ is usually employed, organometallic precursors. Monodisperse CdSe achieved through organometallic reagents' pyrolysis between $250{ }^{\circ} \mathrm{C}$ to 300 ${ }^{\circ} \mathrm{C}$ by injecting in a hot coordinating liquid [69-71]. Different sizes of QDs formed relying upon the states of temperature. The organometallic method is right now regarded as the most vital technique to fabricate QDs. In this technique, various QDs demonstrating high quantum yield can be formed, and QDs size distribution is simply controlled by varying the reaction time or changing the temperature [72-74].

\section{Water phase method/Aqueous solution method}

Ionic perchlorates are utilized as precursors in this method. Ligands like Hydrosulfyl-containing materials, glutathione (GSH), 3mercaptopropionic acid (3-MPA), in an aqueous medium used to prepare CdTe quantum dots. It is an eco-friendly and economical method. Likewise, the QDs formulated by the technique can be straightly given into the biological system. As compared with QDs created from the organometallic process QDs with thiol cap depicted wide size distribution and low quantum yield, poor stability in aqueous solution [75-80].

\section{Hydrothermal method}

QDs with narrow size and high quantum yields were formed by this method. This method also decreases the surface defects which were created during the synthesis process. In this method, the reagents are initially added to the hermetic container and increasing the temperature till supercritical temperature, a high pressure developed with this temperature leading to a successful reduction in the surface defects and reaction time of QDs [8184].

\section{Microwave-assisted method}

This method was initially presented by the Kotov group and pursued by Qian and colleagues. Microwave irradiation as the heating source and water as a solvent were utilized. Heat the reaction system more than $100{ }^{\circ} \mathrm{C}$ to obtained uniform QDs and acquired a quantum yield of $17 \%$. The organic phase synthesis formed hydrophobic QDs. But the QDs must have water solubility for the biological application. The nanocrystals produced from the water solubilization method will remain stable in biological systems and with no effect/change in the photophysical properties. Low fluorescence and less quantum yield achieved with water-soluble QDs formed in the early stage. Afterward, water-soluble QDs provide a higher quantum yield up to $50 \%$ with surface modifications and advanced synthetic procedures $[85,86]$. But the huge challenge is to control the chemical and photophysical properties of QDs in water. Various methods have been established to formulate water-soluble QDs having smaller particle sizes. Ligand exchange is one method in which hydrophilic groups on QDs surface will exchange with hydrophobic groups through a ligand exchange mechanism. This method is merely using monodentate ligands; it will affect fluorescence efficiency and cause aggregation of nanoparticles. Few vacant sites were left by ligands while detaching from the surfaces, and these vacant sites work as trap centers and produce an accumulation of nanoparticles [87, 88].

This issue can be tackled utilizing ligand cross-linking and the di-thio group rather than the mono-thio group. One more method for developing water-soluble QDs is through the amphiphilic molecule incorporation, for example, phospholipids or polymers [89, 90]. The native surface of QDs ligand in the polymer encapsulation layer does not change. In the long-chain polymer molecules, the increased number of hydrophilic groups enhances the QDs dispersibility in biological buffer solutions and for conjugations of bio-probes gives chemical functional groups. The biocompatibility of QDs-polymer nanocomposites enhances and decreases their cytotoxicity due to the polymers coated on QDs. The QDs probe's photophysical properties vary with the size, structure, and chemical composition of the inorganic nanoparticle utilized for the QDs probe's development. The nanoparticle does not interact because of the lack of biological functioning with biological systems. The biomolecules like nucleic acids and proteins provide interaction with living cells used to decorate inert QDs for better biological application. QDs become more bio-compactable with living systems by incorporation of biomolecules [91-93]. The steps and design criteria used in QDs probes manufacturing are described in fig. 4 [12]. 


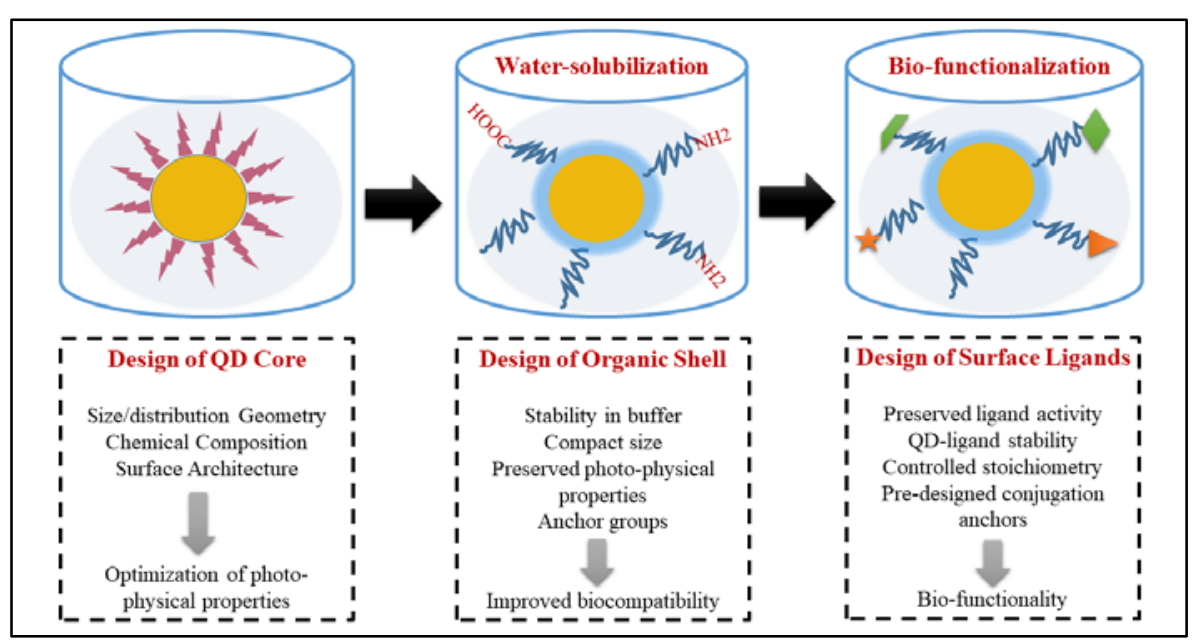

Fig. 4: The steps and design criteria used in QDs probes manufacturing

\section{Surface modifications and Bio-conjugation of QDs}

It is usually observed that the bare QDs are not practical for biological applications because of numerous reasons. Initially, in most of the synthesis approaches, QDs are water-insoluble. Thus, to obtain results from the optical properties of QDs, the surface has to be coated using a hydrophilic agent. Secondly, the core of QDs is very reactive, and with macromolecules, it undergoes potent unspecific interactions resulting in aggregation of particles and variation in fluorescence $[94,95]$. Thirdly, the QDs toxicity can be significantly reduced due to the process of surface modification. The QDs have a hydrophobic surface modified by using hydrophilic agents to solubilize them in aqueous buffers. The peptides, dendrons, or oligomeric phosphines, thiol-containing molecules can be replaced with the hydrophobic surface ligands [96-98].

Further approaches employ phospholipids for modification or hydrophobic interactions between the QDs and amphiphilic polymers or encapsulate the QDs in a silica shell. Numerous potential outcomes are there in which biomolecules can be attached to the QDs. The utilization of QDs containing streptavidin is typically utilized because it is easy to link them to biotin-tagged biomolecules $[99,100]$. Numerous strategies can be used to get QDs bioconjugation consisting of covalent bond formation, multivalent chelation, or passive adsorption. There are two famous cross-linking reactions; active ester maleimide mediated amine, sulfhydryl coupling, and carbodiimide mediated amide formation. The bioconjugation also determines by the hydrophilic coating as the required functional groups deliver by it and should be considered [101-103]

\section{Molecules attachment for targeting}

To the biomolecules like aptamers, small molecule ligands, or antibodies, the QDs must be cross-linked to make them precise to biological targets and thus employed for targeted drug delivery and diagnosis. QDs functionalization can be done by replacing biomolecules with a protein or sulfhydryl group by thiol and peptides with cysteine residues [104-107]. The QDs surface equilibrates with the thiols after incubation, and the biomolecules partially substitute the initial coating molecule. Coating of the QDs with mercapto acid can produce stable covalent bonds. Moreover, the electrostatic interactions among the macromolecules like peptides or proteins and the QDs surface can also give a facial way for modification and coating. Numerous organic functionalities are promptly attached for the QDs encapsulated in a silica shell using well-developed silane chemistry. At present different affinity reagents for QDs modification, like aptamers, small molecules, and peptides, which on cancer cells primarily identify particular overexpressed biomarkers, have been described to manufacture target drug delivery vesicles and cancer diagnosis probes $[16,64$, 108-112].

\section{Characterization of QDs}

Important information regarding the morphology, structure, and physiochemical changes because of conjugation reactions can be disclosed by QDs optical characterization [113, 114]. For optical characterizations of QDs, UV-Vis and photoluminescence spectroscopy are the most utilized, non-destructive, quick, and contactless [115]. The size of QDs is finding out by employing atomic force microscopy, scanning tunneling microscopy, scanning electron microscopy, dynamic light scattering, transmission electron microscopy (TEM) studies [115-117]. The use of Raman scattering spectroscopy and photoluminescence excitation are also reported to determine the size and composition of QDs [118].

\section{Stability of QDs}

In aqueous conditions, a vital role in deciding the stability of QDs depends on the organic molecules and shell attached to the QDs surface. Finally, QDs precipitate with loss in their luminescence because of the loss of surface ligands. The stability of surface ligands can be affected by various processes. It was revealed that common surface ligands used and their photooxidation can cause desorption of hydrophilic thiols by their protonation [113]. To the cadmium chalcogenide QDs deprotonated thiols, thiolates are bound; if the QDs-ligand interface $\mathrm{pH}$ turns down to a particular value, protonation of ligand occurs and remove from the surface of QDs [2, $49,102,119]$. Generally, in a low pH range, the hydrophilic thiols coated QDs precipitated in a pH range of 2 and 7, based on the chemical composition and the size of QDs $[2,45,66,67]$.

The photophysical properties and stability of QDs within the cells firmly subject to the local environment and intracellular localization. Its UV illumination over a long time leads to the photooxidation of surface ligands [46, 47, 65]. Enhancing the packing density and thickness of the ligand shell can hinder the beginning of photooxidation. The core of QDs resists photodegradation because of its inorganic nature, but the core integrity is influenced by the extended UV light exposure $[86,88,91]$.

\section{Cytotoxicity of QDs}

In human beings, cadmium's half-life is around $20 \mathrm{y}$ and is supposed to be carcinogenic, which can deposit in the kidney, liver, and biodistribution in all tissues [120]. The toxicity of QDs relies on numerous chemicals, environmental and physical factors such as size, surface coating, charge, chemical composition, concentration, and free radical production, which might be deciding components of short and long-term in vivo side effects QDs [121]. The particles' biocompatibility has to be described, as QDs are utilized for studies including living cells and organisms [122].

QDs have not been characterized concerning their toxicity because they are new materials. The majority of the QDs contain cadmium, 
which causes lethal impacts when it comes in contact with cells. Efforts need to be taken to secure the core of QDs, by developing a much lower toxicity shell, most of the time ZnS [123], which brought about a marked decrease in cytotoxic effects. The optical properties also enhance this layer; in most biological applications, core-shell systems have turned into the standard. But, to resolve the issue of cytotoxicity completely, the introduction of capping layers was still not adequate. A variety of other factors, such as the particle aggregation on the surface of the cell and even the QDs surface ligands stabilization, have been revealed to affect the cell's viability. The number of factors decides the degree of cytotoxicity, including size, dose, color, capping materials, surface chemistry, and processing parameters $[124,125]$. So far, less information on QDs toxicity, particularly for in vivo use, is accessible. In this manner, QDs toxicity is a fundamental restriction for their utilization. Roberts reported that after administering CdSe/ZnS QDs, it caused lung injury and inflammation in rats. Likewise, Ho et al. detected granuloma following cadmium-based QDs administration in the lung of mice $[123,126,127]$.

\section{Biomedical applications of QDs}

\section{Cell imaging}

Recently as an optional to organic dyes, QDs have been utilized for bioimaging. Furthermore, QDs have a broad absorption spectrum that makes easy excitation of two or multi-photon, particularly in the nearinfrared range. Among all newly proposed imaging strategies, the bioimaging acknowledged by the confocal multi-photonic excitation system is by all accounts the best solution utilizing QDs as fluorescent labels. In this imaging technique, the relatively low excitation energy is harmless for the samples. The QDs' resistance to photobleaching and bright photoluminescence allows even their real-time and long-term imaging. Recently, bioimaging of cancer cells can be done by utilizing QDs as bi photonic fluorescent probes. Based on fluorescence imaging and magnetic resonance imaging (MRI) techniques, a multimodal tumor imaging system was given by Tan et al. [128]. Liu et al. have also fabricated other MRI nanoprobes and bimodal fluorescence with improved fluorescence and can be utilized for labeling HeLa cells [129]. The strong positive MRI contrast was used to characterize these QDs.

\section{Bio-sensing}

A new class of nanomaterials is luminescent semiconductor nanocrystals, the unique generation of fluorescent biosensors are created with the help of specific photophysical properties. Hence, the most common techniques utilized depend on fluorescence changes monitoring in immunoassays for the detection of biomolecules. The fluorescent probes have depicted extensive biosensing applications, diagnostic and clinical assays, ion detection, nucleic acid detection. Generally, the decline in QDs fluorescence emission intensity is seen in the presence of an analyte. For the detection of heavy metal ions and toxic organic substances, fluorescent nanocrystals are usually used. The L-cysteine-capped CdTe QDs synthesis and their utilization in selective trinitrotoluene recognition as the fluorescent probe was reported Chen et al. [130].

\section{Photodynamic therapy}

In the most recent couple of years, the photodynamic treatment (PDT) has come into sight as an advanced technique for malignancy treatment. PDT is an effective alternative to antibiotic therapy. PDT consists of a light, molecular oxygen, and photoactivable agent called photosensitizer (PS). Because of its specificity, PDT is a promising technique, simply the affected cells are exceptionally close to the PS, and until illuminated, the PS isn't cytotoxic. In photodynamic cytotoxicity, through this mechanism, the in situ generations of singlet oxygen (102) seem to assume a crucial role because of the exceedingly productive interaction of the biomolecules with the 102 species [131].

\section{Multiplex coding}

For applications demanding high throughput analysis of biomolecules, multiplex coding has tremendous potential. The small polymer multiplexed beads are embedded inside a finely controlled ratio, which provides a unique optical code. In contrast, oligonucleotides or antibodies act as molecular identifiers, conjugated to the beads' surface $[132,133]$. The intensity levels and types of fluorophores are the two variables that decide the maximum order of multiplexing possible. For instance, two fluorophores provide nine different microbeads with three intensity levels $(1: 1,1: 2, ., 3: 2,3: 3)$. Prior efforts utilized organic fluorophores at multiplexed coding. Spectral overlaps occurred due to their broad emission spectra, and the number of fluorophores that could be used was constrained to only 2-3 dyes, leading to low multiplexing orders [134]. QDs proved to be an ideal replacement for this application because of their narrow and Gaussian-shaped emission spectra. For example, $\mathrm{Xu}$ et al. utilized QDs embedded microspheres to demonstrate the reliability and accuracy of polymerase chain reaction (PCR)-based single nucleotide polymorphism (SNP) genotyping assay $[135,136]$.

\section{Bio-imaging of live cell}

Live-cell imaging is a tough assignment compared with fixed cells and tissues because the thought that must be taken to keep cells alive and delivery of probes across the plasma membrane for examining intracellular targets is the challenging task. For the labeling of cell surface antigens, in vivo use of QDs have been shown. In recent times, the genuine favorable circumstances of QDs have been demonstrated for live-cell imaging by labeling plasma membrane receptors, for example, erbB/HER receptors and glycine receptors enabling single imaging molecules and real-time tracking of biomarkers [137-139]. The information gives new bits of knowledge into the mechanism of the ligand-receptor interaction. For delivery of QDs into the cells, different mechanisms have been utilized, for example, conjugation of QDs to cationic peptides or translocating proteins, or specific membrane receptors, via endocytosis non-specific uptake, and microinjection. Every one of these methods has effectively conveyed QDs into cells, even though it appears that the peptide-mechanism might be the most proficient [140-142].

\section{DNA and RNA detection}

QDs have become an essential tool because of resilient photoluminescence and multiple color properties for multiplexed detection of different DNA targets $[138,143,144]$. For DNA detection FRET technique has been one of the well-known methods. An enzyme is utilized to propel the detection sensitivity and to amplify the target. Excluding FRET, with the help of a magnetic nanoparticletagged DNA probe and a QDs labeled DNA probe with the DNA focus across hybridization detection, could be carried out [139, 145-148].

\section{Gene delivery}

For gene therapy, QDs depicted satisfactory performance as a promising candidate. RNA interference (RNAi) is an encouraging method with an extraordinary chance to battle disease. As RNase quickly degrades siRNAs, they have a short half-life, and they cannot cross the cell membrane because of their negative charge. For the loading of gene molecules, the fluorescent QDs have been examined as potential carriers among a large number of nanoparticles. The positively charged liposomes or polymers suppressed the negative charge of the nucleic acids. Using QDs liposome and QDs-polymer conjugates, imaging of siRNA transfection has been done successfully [149-151]. In a study performed by Lin et al. [152], reported that for silencing and efficient gene delivery, cadmium sulphoselenide/zinc sulfide quantum dots-polyethyleneimine (CdSSe/ZnS QDs-PEI) was found to be a proficient nanoplex formulation.

\section{Plant bio-imaging}

There is expanding use of QDs as markers in plant science for the cells or cell wall. For external agents, the cell wall is the first target location in a plant cell. Djikanovic et al. revealed that in the cell wall of the Picea omorika branch CdSe QDs usually attach to lignin and cellulose. Likewise, by interaction with the chains of $\mathrm{C}=\mathrm{C}$ and $\mathrm{C}-\mathrm{C}$ alternating bonds and interaction with the $\mathrm{OH}$ groups, binding to cellulose and lignin are accomplished $[153,154]$. Data demonstrated that for the homogenous marking of the whole cell wall, the QDs are suitable, which is an outcome of the cell wall polymers that are structurally arranged inside the whole cell wall network. Inside the polymer structures in the cell wall composite, these qualities make possible a feasible nanoparticle penetration $[155,156]$. 


\section{QDs as antimicrobial agents}

The various inorganic nanoparticles having antimicrobial activity have been tried. These comprised a range of metals and their oxide nanoparticles, such as zinc oxide, titanium dioxide, etc. Although upon excitation, these work on the principle of generating reactive oxygen species utilizing high wavelength lights, size-dependent optical properties are an additional benefit of QDs [157]. QDs are known to generate free radicals upon irradiation, and the core material of the semiconductor determined its quality. To the microbes, these produced free radicals are described as toxic, and also, in the process of irradiation, the liberated free heavy-metal ions are harmful to the bacteria [157-159]. Lu et al. depicted that excellent antimicrobial activity possessed by CdTe QDs. In their research, it was also shown that after binding to the bacteria's surface, the QDs disturbs the cellular antioxidative systems, reduces antioxidative enzyme activities, and instigate down-regulation of antioxidative genes [158].

\section{In diagnosis and treatment of cancer}

In the past several decades, cancer has attracted substantial research interest as a leading cause of human death [131, 143]. Ongoing advancements in QDs innovation have effectively had a tremendous effect on cancer imaging.

\section{Sentinel lymph node mapping}

The first lymph node or groups of nodes are the sentinel lymph nodes (SLNS) to which a primary tumor gives metastasizing cancer cells. A method that allows the recognition and the SLNs removal is known as SLN biopsy. Further lymph-node sampling is avoided if cancer is not found in the SLNs. At present, for breast cancer and melanoma staging and prognosis, SLN biopsy is in routine clinical use. Precise nodal mapping is required for success in SLN biopsy. Current mapping methods consist of utilizing peritumoral injection of radioisotopes, for example, the isosulfan blue dye, Technetium$99 \mathrm{~m}$-colloidal albumin, or the combination of the two agents. Kim et al. prepared the type II QDs and coated them with polydentate phosphine making soluble and stable QDs in serum [160]. It was observed that both in pigs and mice, the QDs were promptly identified in nearby SLN after intradermal injection. The multiple QDs under a single excitation source with different emission wavelengths may be used to identify and sort the complicated lymphatic system [161, 162].

\section{Primary tumor detection}

Prostate-specific membrane antigens (PSMA) for cancer imaging may serve as markers or targets for diagnosis. In principle, conjugating the QDs to antibodies, particularly for these antigens, helps the primary tumor recognition and identification of distant or regional metastases. Nie's group has performed some original work utilizing conjugated antibody QDs to target prostate tumor. To nude mice bearing prostate tumor xenografts, a specific PSMA antibody conjugated with $\mathrm{Cd} / \mathrm{Se}$ QDs is intravenously administered. Localization of the QDs to the tumor was seen after $2 \mathrm{~h}$ of circulations [163, 164]. On the other hand, at 15 times higher concentration as compared to conjugated PSMA antibody QDs, when the PEG-modified QDs injected, only after $24 \mathrm{~h}$ circulation reached the tumor presumably by diffusion, a much longer time interval $[165,166]$.

An efficient lymphatic drainage system lacks in the tumor, and blood vessels are leaky; hence at the tumor site, the passive diffusion occurred because of enhanced permeability and retention of QDs. In the clinical setting, to accomplish a maximal impact on cancer diagnosis, research must be progress in the direction of small tumor identification and localization, which are currently not detected through conventional imaging methods [167-169].

\section{QDs as drug nano-carriers}

In the previous decade, with the development of surface modification technique, QDs with water-soluble capping stabilizer, for example, polyethylene glycol polymer, mercaptoethylamine, and mercaptoacetic acid are conjugate promptly with drug molecules through electrostatic interaction or covalent bonds, giving complex nanomedicine with QDs as drug carriers [8, 14, 22, 24].

\section{Other applications}

QDs have additionally been observed to be valuable in the study of microorganisms. Kloepfer et al. revealed that fungi and bacterial glycoproteins could be targeted with QDs conjugates [170]. Numerous different pathogens such as Salmonella Typhi, Giardia lamblia and Cryptosporidium parvum, and Listeria monocytogenes were targeted using QDs [157-159].

Additionally, for Escherichia coli, the QDs were utilized as cell membrane permeable indicators. It might be possible that QDs surface graft with the therapeutic enzymes and activate them producing free radicals (for example, singlet oxygen) or by light by optically cycling the QDs. For determining the content of spironolactone in the tablet, the first applications of QDs were exhibited by Liang and colleagues [171]. In neuroscience research, QDs stand for a new device of enormous potential. The studies that are restricted by the limited anatomy of neuronal and glial interactions are helpful. Photostability, multiplexing potential and inorganic nature are the features of QDs that make them significant worth for drug discovery $[8,11,15,21]$. Outstanding review articles are available in the literature for additional information on QDs fundamentals and applications.

\section{CONCLUSION}

In nanomedicine, the potential applications of QDs span the areas of nano-diagnostics, drug delivery, therapy, and imaging. In the last place, the most promising applications are intracellular imaging, tissue imaging, multiplexed diagnostics, tumor detection, infectious agent detection, immunohistochemistry, and fluoro-immunoassays. Despite all their potential in nanomedicine, QDs are still away from large-scale utilization because of toxicity concerns, regulatory and commercialization issues. The QDs-based bio-nanotechnology will always be a growing list of amazing applications with progress being made in specialized nanoparticle development and the detection of elegant conjugation methods, discovering new targeting ligands.

\section{LIST OF ABBREVIATIONS}

QDs: Quantum Dots; 3-MPA: 3-mercaptopropionic acid; TEM: Transmission Electron Microscopy; MRI: Magnetic Resonance Imaging; PDT: Photodynamic Treatment; PS: Photosensitizer; $10_{2}$ : Singlet Oxygen; PCR: Polymerase Chain Reaction; SNP: Single Nucleotide Polymorphism; RNAi: RNA Interference; SLNs: Sentinel Lymph Nodes; PSMA: Prostate-specific Membrane Antigens

\section{ACKNOWLEDGEMENT}

\section{None}

\section{FUNDING}

Nil

\section{AUTHORS CONTRIBUTIONS}

SCS: conceptualization and literature survey; SGS: literature survey on optical properties of QDs and writing; SDT: a literature review on methods of preparation of QDs and article writing; FSM: literature survey on applications of QDs and writing; SLK: overall literature survey, manuscript writing and referencing; PST: proofreading and contributed in writing style. All authors read and approved the final manuscript.

\section{CONFLICT OF INTERESTS}

The authors declare that they have no competing interests.

\section{REFERENCES}

1. Matea CT, Mocan T, Tabaran F, Pop T, Mosteanu O, Puia C, et al. Quantum dots in imaging, drug delivery and sensor applications. Int J Nanomed 2017;12:5421-31.

2. Moreels I, Justo Y, Geyter B De, Haustraete K, Martins JC, Hens Z. Quantum dots: a surface chemistry study. ACS Nano 2012;5:2004-12. 
3. Dabbousi BO, Rodriguez Viejo J, Mikulec FV, Heine JR, Mattoussi $\mathrm{H}$, Ober R, et al. (CdSe)ZnS core-shell quantum dots: synthesis and characterization of a size series of highly luminescent nanocrystallites. J Phys Chem B 1997;101:9463-75.

4. Maxwell T, Nogueira Campos MG, Smith S, Doomra M, Thwin Z, Santra S. Quantum dots. In: Nanoparticles for biomedical applications: fundamental concepts, biological interactions and clinical applications; 2019. p. 243-65.

5. Senthil Kumar M, Valarmathi S, Bhima P, Prudhvi Devabaktuni S, Raja A, Vallabhaneni SD. Quantum dots. Int J Pharm Technol 2012;4:1929-49.

6. Bawendi MG, Steigerwald ML, Brus LE. The quantum mechanics of larger semiconductor clusters ("Quantum dots"). Annu Rev Phys Chem 1990;41:477-96.

7. Biju V. Chemical modifications and bioconjugate reactions of nanomaterials for sensing, imaging, drug delivery and therapy. Chem Soc Rev 2014;43:744-64.

8. Portney NG, Ozkan M. Nano-oncology: drug delivery, imaging, and sensing. Anal Bioanal Chem 2006;384:620-30.

9. Godbole NN, Galgatte UC, Chaudhari PD. Development and in vitro evaluation of quantum dots as a carrier for delivery of 5fluorouracil. Int J Pharm Pharm Sci 2016;8:289-96.

10. SLJ, Gupta NV. Diabetic retinopathy: an inclusive review on current treatment and management approaches. Asian J Pharm Clin Res 2018;11:54.

11. Qi L, Gao X. Emerging application of quantum dots for drug delivery and therapy. Expert Opinion Drug Delivery 2008;5:263-7.

12. Zrazhevskiy $P$, Sena $M$, Gao $X$. Designing multifunctional quantum dots for bioimaging, detection, and drug delivery. Chem Soc Rev 2010;39:4326-54.

13. Maysinger D, Lovric J, Eisenberg A, Savic R. Fate of micelles and quantum dots in cells. Eur J Pharm Biopharm 2007;65:270-81.

14. Caruthers SD, Wickline SA, Lanza GM. Nanotechnological applications in medicine. Curr Opinion Biotechnol 2007;18:26-30.

15. Wagner AM, Knipe JM, Orive G, Peppas NA. Quantum dots in biomedical applications. Acta Biomaterialia 2019;94:44-63.

16. Kong FY, Zhang JW, Li RF, Wang ZX, Wang WJ, Wang W. Unique roles of gold nanoparticles in drug delivery, targeting and imaging applications. Molecules 2017;22:1445.

17. Wang $Q$, Huang $X$, Long $Y$, Wang $X$, Zhang $H$, Zhu $R$, et al. Hollow luminescent carbon dots for drug delivery. Carbon 2013;59:192-9.

18. Wegner KD, Hildebrandt N. Quantum dots: Bright and versatile in vitro and in vivo fluorescence imaging biosensors. Chem Soc Rev 2015;44:4792-834.

19. Lee JH, Yigit MV, Mazumdar D, Lu Y. Molecular diagnostic and drug delivery agents based on aptamer-nanomaterial conjugates. Adv Drug Delivery Rev 2010;62:592-605.

20. Xu G, Zeng S, Zhang B, Swihart MT, Yong KT, Prasad PN. New generation cadmium-free quantum dots for biophotonics and nanomedicine. Chem Rev 2016;116:12234-327.

21. Chinnathambi S, Chen S, Ganesan S, Hanagata N. Silicon quantum dots for biological applications. Adv Healthcare Mater 2014;3:10-29.

22. Probst CE, Zrazhevskiy P, Bagalkot V, Gao X. Quantum dots as a platform for nanoparticle drug delivery vehicle design. Adv Drug Delivery Rev 2013;65:703-18.

23. Frecker T, Bailey D, Arzeta Ferrer X, McBride J, Rosenthal SJ. Review-quantum dots and their application in lighting, displays, and biology. ECS J Solid State Sci Technol 2016;5:R3019-31.

24. Lin J, Chen X, Huang P. Graphene-based nanomaterials for bioimaging. Adv Drug Delivery Rev 2016;105:242-54.

25. Ramamurthy SK, Sridhar C. Parthenium mediated synthesis of zinc oxide nanoparticles and its characterization. Int J Appl Pharm 2019;11:113-6.

26. Sivapriya V, Ponnarmadha S, Azeezand NA, Sudarshanadeepa V. Novel nanocarriers for ethnopharmacological formulations. Int J Appl Pharm 2018;10:26-30.

27. Jinhao GAO, Hongwei GU, Bing XU. Multifunctional magnetic nanoparticles: design, synthesis, and biomedical applications. Acc Chem Res 2009;42:1097-107.

28. Cassette E, Helle M, Bezdetnaya L, Marchal F, Dubertret B, Pons T. Design of new quantum dot materials for deep tissue infrared imaging. Adv Drug Delivery Rev 2013;65:719-31.
29. Martinez Carmona M, Gun'Ko Y, Vallet Regi M. Zno nanostructures for drug delivery and theranostic applications. Nanomaterials 2018;8:268.

30. Boakye Yiadom KO, Kesse S, Opoku Damoah Y, Filli MS, Aquib $\mathrm{M}$, Joelle MMB, et al. Carbon dots: applications in bioimaging and theranostics. Int J Pharm 2019;564:308-17.

31. Liu ML, Chen B Bin, Li CM, Huang CZ. Carbon dots: synthesis, formation mechanism, fluorescence origin and sensing applications. Green Chem 2019;21:449-71.

32. Delehanty JB, Mattoussi H, Medintz IL. Delivering quantum dots into cells: Strategies, progress and remaining issues. Anal Bioanal Chem 2009;393:1091-105.

33. Bilan R, Fleury F, Nabiev I, Sukhanova A. Quantum dot surface chemistry and functionalization for cell targeting and imaging. Bioconjugate Chem 2015;26:609-24.

34. Hild WA, Breunig M, Goepferich A. Quantum dots-nano-sized probes for the exploration of cellular and intracellular targeting. Eur J Pharm Biopharm 2008;68:153-68.

35. Bulte JWM, Modo MMJ. Design and applications of nanoparticles in biomedical imaging. Design and applications of nanoparticles in biomedical imaging; 2016. p. 1-469.

36. Azzazy HME, Mansour MMH, Kazmierczak SC. From diagnostics to therapy: prospects of quantum dots. Clin Biochem 2007;40:917-27.

37. Massey M, Wu M, Conroy EM, Algar WR. Mind your P's and Q's: the coming of age of semiconducting polymer dots and semiconductor quantum dots in biological applications. Curr Opinion Biotechnol 2015;34:30-40.

38. Jaleel JA, Pramod K. Artful and multifaceted applications of carbon dot in biomedicine. J Controlled Release 2018;269:302-21.

39. Gittard SD, Miller PR, Boehm RD, Ovsianikov A, Chichkov BN, Heiser J, et al. Multiphoton microscopy of transdermal quantum dot delivery using two-photon polymerization-fabricated polymer microneedles. Faraday Discuss 2011;149:171-85.

40. Zuo P, Lu X, Sun Z, Guo Y, He H. A review on syntheses, properties, characterization and bioanalytical applications of fluorescent carbon dots. Microchim Acta 2016;183:519-42.

41. Ding C, Zhu A, Tian Y. Functional surface engineering of C-dots for fluorescent biosensing and in vivo bioimaging. Acc Chem Res 2014;47:20-30.

42. Homan K, Mallidi S, Cooley E, Emelianov S. Combined photoacoustic and ultrasound imaging of metal nanoparticles in vivo. Nanoimaging 2011;3:225-49.

43. Gao X, Du C, Zhuang Z, Chen W. Carbon quantum dot-based nanoprobes for metal ion detection. J Materials Chem C 2016;4:6927-45.

44. Geszke Moritz M, Moritz M. Quantum dots as versatile probes in medical sciences: synthesis, modification and properties. Mater Sci Eng 2013;33:1008-21.

45. Molaei MJ. A review on nanostructured carbon quantum dots and their applications in biotechnology, sensors, and chemiluminescence. Talanta 2019;196:456-78.

46. Bajwa N, Mehra NK, Jain K, Jain NK. Pharmaceutical and biomedical applications of quantum dots. Artif Cells Nanomed Biotechnol 2016;44:758-68.

47. Dey N, Rao M. Quantum dot: novel carrier for drug delivery. Int J Res Pharm Biomed Sci 2011;2:448-58.

48. Mo D, Hu L, Zeng G, Chen G, Wan J, Yu Z, et al. Cadmiumcontaining quantum dots: properties, applications, and toxicity. Appl Microbiol Biotechnol 2017;101:2713-33.

49. Nakahara M, Ohmi T. Quantum computing with quantum dots. In: Quantum Computing; 2008. p. 377-98.

50. Wu P, Yan XP. Doped quantum dots for chemo/biosensing and bioimaging. Chem Soc Rev 2013;42:5489-521.

51. Biju V, Itoh T, Ishikawa M. Delivering quantum dots to cells: bioconjugated quantum dots for targeted and nonspecific extracellular and intracellular imaging. Chem Soc Rev 2010;39:3031-56.

52. Gaponik N, Talapin DV, Rogach AL, Hoppe K, Shevchenko EV, Kornowski A, et al. Thiol-capping of CDTe nanocrystals: An alternative to organometallic synthetic routes. J Phys Chem B 2002;106:7177-85.

53. Emin S, Singh SP, Han L, Satoh N, Islam A. Colloidal quantum dot solar cells. Sol Energy 2011;85:1264-82. 
54. Chen W, Lv G, Hu W, Li D, Chen S, Dai Z. Synthesis and applications of graphene quantum dots: a review. Nanotechnol Rev 2018;7:157-85.

55. Campuzano S, Yanez Sedeno P, Pingarron JM. Carbon dots and graphene quantum dots in electrochemical biosensing. Nanomaterials 2019;9:634.

56. Lim SY, Shen W, Gao Z. Carbon quantum dots and their applications. Chem Soc Rev 2015;44:362-81.

57. Barroso MM. Quantum dots in cell biology. J Histochem Cytochem 2011;59:237-51.

58. Klimov VI, Mikhailovsky AA, Xu S, Malko A, Hollingsworth JA, Leatherdale CA, et al. Optical gain and stimulated emission in nanocrystal quantum dots. Science 2000;290:314-7.

59. Kim S, Fisher B, Eisler HJ, Bawendi M. Type-II quantum dots: $\mathrm{CdTe} / \mathrm{CdSe}$ (core/shell) and CdSe/ZnTe(core/shell) heterostructures. J Am Chem Soc 2003;125:11466-7.

60. Chan WCW, Maxwell DJ, Gao X, Bailey RE, Han M, Nie S. Luminescent quantum dots for multiplexed biological detection and imaging. Curr Opinion Biotechnol 2002;13:40-6.

61. True LD, Gao X. Quantum dots for molecular pathology: their time has arrived. J Mol Diagnostics 2007;9:7-11.

62. Moloney MP, Govan J, Loudon A, Mukhina M, Gun'ko YK. Preparation of chiral quantum dots. Nat Protoc 2015;10:558-73.

63. Ghaderi S, Ramesh B, Seifalian AM. Fluorescence nanoparticles "quantum dots" as drug delivery system and their toxicity: a review. J Drug Targeting 2011;19:475-86.

64. Nurunnabi M, Parvez K, Nafiujjaman M, Revuri V, Khan HA, Feng X, et al. Bioapplication of graphene oxide derivatives: drug/gene delivery, imaging, polymeric modification, toxicology, therapeutics and challenges. RSC Adv 2015;5:42141-61.

65. Obonyo O, Fisher E, Edwards M, Douroumis D. Quantum dots synthesis and biological applications as imaging and drug delivery systems. Crit Rev Biotechnol 2010;30:283-301.

66. Bilan R, Nabiev I, Sukhanova A. Quantum dot-based nanotools for bioimaging, diagnostics, and drug delivery. Chem BioChem 2016;17:2103-14.

67. Gulia S, Kakkar R. Zno quantum dots for biomedical applications. Adv Materials Lett 2013;4:876-87.

68. Liu X, Pang J, Xu F, Zhang X. Simple approach to synthesize amino-functionalized carbon dots by carbonization of chitosan. Sci Rep 2016;6:31100.

69. Coe S, Woo WK, Bawendi M, Bulovic V. Electroluminescence from single monolayers of nanocrystals in molecular organic devices. Nature 2002;420:800-3.

70. Swarnkar A, Marshall AR, Sanehira EM, Chernomordik BD, Moore DT, Christians JA, et al. Quantum dot-induced phase stabilization of $\alpha$-CsPbI3 perovskite for high-efficiency photovoltaics. Science 2016;354:92-5.

71. Bak S, Kim D, Lee H. Graphene quantum dots and their possible energy applications: a review. Curr Appl Physics 2016;16:1192-201.

72. Grisorio R, Debellis D, Suranna GP, Gigli G, Giansante C. The dynamic organic/inorganic interface of colloidal $\mathrm{PbS}$ quantum dots. Angew Chem Int Ed 2016;55:6628-33.

73. Seguin R, Schliwa A, Rodt S, Potschke K, Pohl UW, Bimberg D. Size-dependent fine-structure splitting in self-organized InAs/GaAs quantum dots. Phys Rev Lett 2005;95:257402.

74. Bhattacharya P, Ghosh S, Stiff Roberts AD. Quantum dot optoelectronic devices. Annu Rev Mater Res 2004;34:1-40.

75. Li H, He X, Kang Z, Huang H, Liu Y, Liu J, et al. Water-soluble fluorescent carbon quantum dots and photocatalyst design. Angew Chem Int Ed 2010;49:4430-4.

76. Darbandi M, Thomann R, Nann T. Single quantum dots in silica spheres by microemulsion synthesis. Chem Mater 2005;17:5720-5.

77. Tang L, Ji R, Cao X, Lin J, Jiang H, Li X, et al. Deep ultraviolet photoluminescence of water-soluble self-passivated graphene quantum dots. ACS Nano 2012;6:5102-10.

78. Wang F, Pang S, Wang L, Li Q, Kreiter M, Liu CY. One-step synthesis of highly luminescent carbon dots in noncoordinating solvents. Chem Mater 2010;22:4528-30.

79. Ahirwar S, Mallick S, Bahadur D. Electrochemical method to prepare graphene quantum dots and graphene oxide quantum dots. ACS Omega 2017;2:8343-53.
80. Nann T. Phase-transfer of CdSe@ZnS quantum dots using amphiphilic hyperbranched polyethyleneimine. Chem Commun 2005;13:1735-6.

81. Tian P, Tang L, Teng KS, Lau SP. Graphene quantum dots from chemistry to applications. Materials Today Chem 2018;10:221-58.

82. Baruah S, Dutta J. Hydrothermal growth of $\mathrm{ZnO}$ nanostructures. Sci Technol Adv Mater 2009;10:013001.

83. Wang Q, Zheng H, Long Y, Zhang L, Gao M, Bai W. Microwavehydrothermal synthesis of fluorescent carbon dots from graphite oxide. Carbon NY 2011;49:3134-40.

84. Prasannan A, Imae T. One-pot synthesis of fluorescent carbon dots from orange waste peels. Ind Eng Chem Res 2013;52:15673-8.

85. Park SY, Thongsai N, Chae A, Jo S, Kang EB, Paoprasert P, et al. Microwave-assisted synthesis of luminescent and biocompatible lysine-based carbon quantum dots. J Ind Eng Chem 2017;47:329-35.

86. He Y, Zhong Y, Peng F, Wei X, Su Y, Lu Y, et al. One-pot microwave synthesis of water-dispersible, ultraphoto-and $\mathrm{pH}$ stable, and highly fluorescent silicon quantum dots. J Am Chem Soc 2011;133:14192-5.

87. Sumanth Kumar D, Jai Kumar B, Mahesh HM. Quantum nanostructures (QDs): an overview. In: Synthesis of Inorganic Nanomaterials; 2018. p. 59-88.

88. Liu Y, Xiao N, Gong N, Wang H, Shi X, Gu W, et al. One-step microwave-assisted polyol synthesis of green luminescent carbon dots as optical nanoprobes. Carbon 2014;68:258-64.

89. Singh I, Arora R, Dhiman H, Pahwa R. Carbon quantum dots: Synthesis, characterization and biomedical applications. Turkish J Pharm Sci 2018;15:219-30.

90. Wang X, Feng Y, Dong P, Huang J. A mini-review on carbon quantum dots: preparation, properties, and electrocatalytic application. Front Chem 2019;7:671.

91. Qian H, Qiu X, Li L, Ren J. Microwave-assisted aqueous synthesis: A rapid approach to prepare highly luminescent $\mathrm{ZnSe}(\mathrm{S})$ alloyed quantum dots. J Phys Chem B 2006;110:9034-40.

92. Rangel Mendez JR, Matos J, Chazaro Ruiz LF, Gonzalez Castillo AC, Barrios Yanez G. Microwave-assisted synthesis of C-doped TiO 2 and ZnO hybrid nanostructured materials as quantumdots sensitized solar cells. Appl Surf Sci 2018;434:744-55.

93. Lehnen T, Zopes D, Mathur S. Phase-selective microwave synthesis and inkjet printing applications of Zn 2SnO 4 (ZTO) quantum dots. J Mater Chem 2012;22:17732-6.

94. Vasudevan D, Gaddam RR, Trinchi A, Cole I. Core-shell quantum dots: properties and applications. J Alloys Compounds 2015;636:395-404.

95. Wu YL, Lim CS, Fu S, Tok AIY, Lau HM, Boey FYC, et al. Surface modifications of $\mathrm{ZnO}$ quantum dots for bio-imaging. Nanotechnology 2007;18:215604.

96. Monopoli MP, Pitek AS, Lynch I, Dawson KA. Nanomaterial interfaces in biology. In: Nanomaterial Interfaces in Biology: Methods and Protocols, Methods in Molecular Biology; 2013;1025:137155.

97. Yuan CT, Chou WC, Chuu DS, Chang WH, Lin HS, Ruaan RC. Fluorescence properties of colloidal CdSe/ZnS quantum dots with various surface modifications. J Med Biol Eng 2006;26:131-5.

98. Krogmeier JR, Kang HG, Clarke ML, Yim P, Hwang J. Probing the dynamic fluorescence properties of single water-soluble quantum dots. Opt Commun 2008;281:1781-8.

99. Kumar P, Kukkar D, Deep A, Sharma SC, Bharadwaj LM. Synthesis of mercaptopropionic acid stabilized CDS quantum dots for bioimaging in breast cancer. Adv Mater Lett 2012;3:471-5.

100. Borse V, Sadawana M, Srivastava R. CdTe quantum dots: aqueous phase synthesis, stability studies and protein conjugation for development of biosensors. In: Nanophotonics VI; 2016;9884:988423.

101. Singla R, Guliani A, Kumari A, Yadav SK. Metallic nanoparticles, toxicity issues and applications in medicine. In: Nanoscale materials in targeted drug delivery, theragnosis and tissue regeneration; 2016. p. 41-80.

102. Dobhal G, Garima. Quantum dot bioconjugates for the detection of extracellular vesicles in saliva and breath. MSc Thesis; 2019. Available from http://researcharchive.vuw.ac.nz/handle/ 10063/8119. [Last accessed on 10 Mar 2021]. 
103. Tian B, Al-Jamal WT, Stuart M, Kostarelos K. Doxorubicinloaded and antibody-conjugated liposome-QD hybrid vesicles for targeted cancer therapy and imaging. In: Nanotechnology 2010: Bio Sensors, Instruments, Medical, Environment and Energy-Technical Proceedings of the 2010 NSTI Nanotechnology Conference and Expo, NSTI-Nanotech 2010; 3:380-1.

104. Pardo J, Peng Z, Leblanc RM. Cancer targeting and drug delivery using carbon-based quantum dots and nanotubes. Molecules 2018;23:378.

105. Howarth M, Takao K, Hayashi Y, Ting AY. Targeting quantum dots to surface proteins in living cells with biotin ligase. Proc Natl Acad Sci USA 2005;102:7583-8

106. Liu W, Howarth M, Greytak AB, Zheng Y, Nocera DG, Ting AY, et al. Compact biocompatible quantum dots functionalized for cellular imaging. J Am Chem Soc 2008;130:1274-84.

107. Smith AM, Duan H, Mohs AM, Nie S. Bioconjugated quantum dots for in vivo molecular and cellular imaging. Adv Drug Delivery Rev 2008;60:1226-40.

108. Ghasemi Y, Peymani P, Afifi S. Quantum dot: magic nanoparticle for imaging, detection and targeting. Acta Biomed l'Ateneo Parm 2009;80:156-65.

109. Kim D, Kim DH, Lee JH, Grossman JC. Impact of stoichiometry on the electronic structure of PbS quantum dots. Phys Rev Lett 2013;110:196802.

110. Rhyner MN, Smith AM, Goo X, Mao H, Yang L, Nie S. Quantum dots and multifunctional nanoparticles: new contrast agents for tumor imaging. Nanomedicine 2006;1:209-17.

111. Liu W, Hak SC, Zimmer JP, Tanaka E, Frangioni JV, Bawendi M. Compact cysteine-coated $\mathrm{CdSe}(\mathrm{ZnCdS})$ quantum dots for in vivo applications. J Am Chem Soc 2007;129:14530-1.

112. Liu DS, Phipps WS, Loh KH, Howarth M, Ting AY. Quantum dot targeting with lipoic acid ligase and HaloTag for singlemolecule imaging on living cells. ACS Nano 2012;6:11080-7.

113. Drbohlavova J, Adam V, Kizek R, Hubalek J. Quantum dotscharacterization, preparation and usage in biological systems. Int J Mol Sci 2009;10:656-73.

114. Murray CB, Kagan CR, Bawendi MG. Self-organization of CdSe nanocrystallites into three-dimensional quantum dot superlattices. Science 1995;270:1335-8.

115. Micic OI, Curtis CJ, Jones KM, Sprague JR, Nozik AJ. Synthesis and characterization of InP quantum dots. J Phys Chem 1994;98:4966-9.

116. Lipovskii A, Kolobkova E, Petrikov V, Kang I, Olkhovets A, Krauss T, et al. Synthesis and characterization of PbSe quantum dots in phosphate glass. Appl Phys Lett 1997;71:3406-8.

117. Knittel F, Gravel E, Cassette E, Pons T, Pillon F, Dubertret B, et al. On the characterization of the surface chemistry of quantum dots. Nano Lett 2013;13:5075-8.

118. Micic OI, Sprague JR, Curtis CJ, Jones KM, Machol JL, Nozik AJ, et al. Synthesis and characterization of InP, GaP, and GaInP2 quantum dots. J Phys Chem 1995;99:7754-9.

119. Passaseo A. Quantum dots: introduction. Science 2010;4:1-25.

120. Derfus AM, Chan WCW, Bhatia SN. Probing the cytotoxicity of semiconductor quantum dots. Nano Lett 2004;4:11-8.

121. Hoshino A, Fujioka K, Oku T, Suga M, Sasaki YF, Ohta T, et al. Physicochemical properties and cellular toxicity of nanocrystal quantum dots depend on their surface modification. Nano Lett 2004;4:2163-9.

122. Zhao F, Zhao Y, Liu Y, Chang X, Chen C, Zhao Y. Cellular uptake, intracellular trafficking, and cytotoxicity of nanomaterials. Small 2011;7:1322-37.

123. Su Y, Hu M, Fan C, He Y, Li Q, Li W, et al. The cytotoxicity of CdTe quantum dots and the relative contributions from released cadmium ions and nanoparticle properties. Biomaterials 2010;31:4829-34.

124. Chong Y, Ma Y, Shen $\mathrm{H}, \mathrm{Tu} \mathrm{X}, \mathrm{Zhou} \mathrm{X}, \mathrm{Xu}$ J, et al. The in vitro and in vivo toxicity of graphene quantum dots. Biomaterials 2014;35:5041-8.

125. Tsay JM, Michalet X. New light on quantum dot cytotoxicity. Chem Biol 2005;12:1159-61.

126. Wu C, Wang C, Han T, Zhou X, Guo S, Zhang J. Insight into the cellular internalization and cytotoxicity of graphene quantum dots. Adv Healthc Mater 2013;2:1613-9.
127. Guo G, Liu W, Liang J, He Z, Xu H, Yang X. Probing the cytotoxicity of CdSe quantum dots with surface modification. Mater Lett 2007;61:1641-4.

128. Tan YF, Chandrasekharan P, Maity D, Yong CX, Chuang $\mathrm{KH}$, Zhao $\mathrm{Y}$, et al. Multimodal tumor imaging by iron oxides and quantum dots formulated in poly (lactic acid)-d-alphatocopheryl polyethylene glycol 1000 succinate nanoparticles. Biomaterials 2011;32:2969-78.

129. Liu Y, Ai K, Yuan Q, Lu L. Fluorescence-enhanced gadoliniumdoped zinc oxide quantum dots for magnetic resonance and fluorescence imaging. Biomaterials 2011;32:1185-92.

130. Chen Y, Chen Z, He Y, Lin H, Sheng P, Liu C, et al. L-cysteinecapped CdTe QD-based sensor for simple and selective detection of trinitrotoluene. Nanotechnology 2010;21:125502.

131. Juzenas P, Chen W, Sun YP, Coelho MAN, Generalov R, Generalova $\mathrm{N}$, et al. Quantum dots and nanoparticles for photodynamic and radiation therapies of cancer. Adv Drug Delivery Rev 2008;60:1600-14.

132. Han M, Gao X, Su JZ, Nie S. Quantum-dot-tagged microbeads for multiplexed optical coding of biomolecules. Nat Biotechnol 2001;19:631-5.

133. Lagerholm BC, Wang M, Ernst LA, Ly DH, Liu H, Bruchez MP, et al. Multicolor coding of cells with cationic peptide coated quantum dots. Nano Lett 2004:4:2019-22.

134. Li J, Zhao XW, Zhao YJ, Gu ZZ. Quantum-dot-coated encoded silica colloidal crystals beads for multiplex coding. Chem Commun 2009;17:2329-31.

135. Tholouli E, Sweeney E, Barrow E, Clay V, Hoyland JA, Byers RJ. Quantum dots light up pathology. J Pathol 2008;216:275-85.

136. Xia Z, Xing Y, So MK, Koh AL, Sinclair R, Rao J. Multiplex detection of protease activity with quantum dot nanosensors prepared by intein-mediated specific bioconjugation. Anal Chem 2008;80:8649-55.

137. Zheng XT, Ananthanarayanan A, Luo KQ, Chen P. Glowing graphene quantum dots and carbon dots: properties, syntheses, and biological applications. Small 2015;11:1620-36.

138. Bourlinos AB, Stassinopoulos A, Anglos D, Zboril R, Karakassides M, Giannelis EP. Surface functionalized carbogenic quantum dots. Small 2008;4:455-8.

139. Kagan CR, Murray CB, Nirmal M, Bawendi MG. Electronic energy transfer in CdSe quantum dot solids. Phys Rev Lett 1996;76:1517-20.

140. Valizadeh A, Mikaeili H, Samiei M, Farkhani SM, Zarghami N, Kouhi M, et al. Quantum dots: synthesis, bioapplications, and toxicity. Nanoscale Res Lett 2012;7:480.

141. Luo PG, Sahu S, Yang ST, Sonkar SK, Wang J, Wang H, et al. Carbon "quantum" dots for optical bioimaging. J Mater Chem B 2013;1:2116-27.

142. Bakker MA, Mehl S, Hiltunen T, Harju A, Divincenzo DP. Validity of the single-particle description and charge noise resilience for multielectron quantum dots. Phys Rev B-Condens Matter Mater Phys 2015;91:155425.

143. Choi YE, Kwak JW, Park JW. Nanotechnology for early cancer detection. Sensors 2010;10:428-55.

144. Walling MA, Novak JA, Shepard JRE. Quantum dots for live cell and in vivo imaging. Int J Mol Sci 2009;10:441-91.

145. Qian ZS, Shan XY, Chai LJ, Ma JJ, Chen JR, Feng H. DNA nanosensor based on biocompatible graphene quantum dots and carbon nanotubes. Biosens Bioelectron 2014;60:64-70.

146. Obliosca JM, Liu C, Batson RA, Babin MC, Werner JH, Yeh HC. DNA/RNA detection using DNA-templated few-atom silver nanoclusters. Biosensors 2013;3:185-200.

147. Su S, Fan J, Xue B, Yuwen L, Liu X, Pan D, et al. DNA-conjugated quantum dot nanoprobe for high-sensitivity fluorescent detection of DNA and micro-RNA. ACS Appl Mater Interfaces 2014;6:1152-7.

148. Tikhomirov G, Hoogland S, Lee PE, Fischer A, Sargent EH, Kelley SO. DNA-based programming of quantum dot valency, self assembly and luminescence. Nat Nanotechnol 2011;6:485-90.

149. Kim SS, Ye C, Kumar P, Chiu I, Subramanya S, Wu H, et al. Targeted delivery of sirna to macrophages for antiinflammatory treatment. Mol Ther 2010;18:993-1001.

150. Bonoiu A, Mahajan SD, Ye L, Kumar R, Ding H, Yong KT, et al. MMP-9 gene silencing by a quantum dot-siRNA nanoplex 
delivery to maintain the integrity of the blood brain barrier. Brain Res 2009;1282:142-55.

151. Bruun J, Larsen TB, Jølck RI, Eliasen R, Holm R, Gjetting T, et al. Investigation of enzyme-sensitive lipid nanoparticles for delivery of siRNA to blood-brain barrier and glioma cells. Int ] Nanomed 2015;10:5995-6008.

152. Lin G, Chen T, Zou J, Wang Y, Wang X, Li J, et al. Quantum dotssiRNA nanoplexes for gene silencing in central nervous system tumor cells. Front Pharmacol 2017;8:182.

153. Djikanovic D, Kalauzi A, Jeremic M, Xu J, Micic M, Whyte JD, et al. Interaction of the CdSe quantum dots with plant cell walls. Colloids Surf B 2012;91:41-7.

154. Wang J, Yang Y, Zhu H, Braam J, Schnoor JL, Alvarez PJJ. Uptake, translocation, and transformation of quantum dots with cationic versus anionic coatings by Populus deltoides $\times$ nigra cuttings. Environ Sci Technol 2014;48:6754-62.

155. Zhang D, Hua T, Xiao F, Chen C, Gersberg RM, Liu Y, et al. Uptake and accumulation of $\mathrm{CuO}$ nanoparticles and $\mathrm{CdS} / \mathrm{ZnS}$ quantum dot nanoparticles by schoenoplectus tabernaemontani in hydroponic mesocosms. Ecol Eng 2014;70:114-23.

156. Wang Q, Chen J, Zhang H, Lu M, Qiu D, Wen Y, et al. Synthesis of water-soluble quantum dots for monitoring carrier-DNA nanoparticles in plant cells. J Nanosci Nanotechnol 2011;11:2208-14.

157. Dong X, Liang W, Meziani MJ, Sun YP, Yang L. Carbon dots as potent antimicrobial agents. Theranostics 2020;10:671-86.

158. Lu Z, Li CM, Bao H, Qiao Y, Toh Y, Yang X. Mechanism of antimicrobial activity of CdTe quantum dots. Langmuir 2008;24:5445-52.

159. Ristic BZ, Milenkovic MM, Dakic IR, Todorovic Markovic BM Milosavljevic MS, Budimir MD, et al. Photodynamic antibacterial effect of graphene quantum dots. Biomaterials 2014;35:4428-35.
160. Kim S, Lim YT, Soltesz EG, De Grand AM, Lee J, Nakayama A, et al. Near-infrared fluorescent type II quantum dots for sentinel lymph node mapping. Nat Biotechnol 2004;22:93-7.

161. Frangioni JV, Kim SW, Ohnishi S, Kim S, Bawendi MG. Sentinel lymph node mapping with type-II quantum dots. Methods Mol Biol 2007;374:147-59.

162. Pons T, Pic E, Lequeux N, Cassette E, Bezdetnaya L, Guillemin F, et al. Cadmium-free CuInS2/ZnS quantum dots for sentinel lymph node imaging with reduced toxicity. ACS Nano 2010;4:2531-8.

163. Smith AM, Dave S, Nie S, True L, Gao X. Multicolor quantum dots for molecular diagnostics of cancer. Expert Rev Mol Diagnostics 2006;6:231-44.

164. Shao L, Gao Y, Yan F. Semiconductor quantum dots for biomedical applications. Sensors 2011;11:11736-51.

165. Luo G, Long J, Zhang B, Liu C, Ji S, Xu J, et al. Quantum dots in cancer therapy. Expert Opinion Drug Delivery 2012;9:47-58.

166. Nie S, Xing Y, Kim GJ, Simons JW. Nanotechnology applications in cancer. Annual Rev Biomed Eng 2007;9:257-88.

167. Wagner MK, Li F, Li J, Li XF, Le XC. Use of quantum dots in the development of assays for cancer biomarkers. Anal Bioanal Chem 2010;397:3213-24.

168. Nida DL, Rahman MS, Carlson KD, Richards Kortum R, Follen M. Fluorescent nanocrystals for use in early cervical cancer detection. Gynecol Oncol 2005;99(3 Suppl 1):S89-94.

169. Iga AM, Robertson JHP, Winslet MC, Seifalian AM. Clinical potential of quantum dots. J Biomed Biotechnol 2007;2007:76087.

170. Kloepfer JA, Mielke RE, Wong MS, Nealson KH, Stucky G, Nadeau JL. Quantum dots as strain-and metabolism-specific microbiological labels. Appl Environ Microbiol 2003;69:4205-13.

171. Liang J, Huang S, Zeng D, He Z, Ji X, Ai X, et al. CdSe quantum dots as luminescent probes for spironolactone determination. Talanta 2006;69:126-30. 